\title{
A Novel Approach for Automatic Detection of Defects in Radiographic Weld Images by Morphological and Statistical Operations
}

\author{
Dr. T. Srikanth ${ }^{1}$, Dr. V.Kamala ${ }^{2}$ \\ ${ }^{I}$ (Professor, Dept. of Mech. Engg., VSM COE, Ramachandrapuram, A.P., India) \\ ${ }^{2}(D G M($ Retd.), BHEL, R\&D, Balanagar, Hyderabad, A.P., India)
}

\begin{abstract}
In this paper a novel approach for detecting the defects in weld radiographs is presented by using combinatorial morphological and statistical operations. The proposed method consists of several stages. The procedure first involves the application of wiener for noise removal and enhancing the original image. Then, the morphological opening operation is applied to find the fine interior segments. In the third step, the defects in the weld image are obtained by applying statistical operations. And finally, the global threshold operation is used to convert the resultant gray level image to binary image. The proposed novel method is applied on various radiographic weld images and the results obtained show the validity of the proposed method.
\end{abstract}

Keywords - Dilation, Erosion, Segmentation, Weld defect, Wiener filter.

\section{INTRODUCTION}

In evaluating the results of radiographic testing, manual work is often adopted in manufacture enterprises. This evaluating process accompanies a large amount of workloads but low efficiency, and is harmful to the specialists, whose experience and mood to his work may directly influence the accuracy of the evaluation. Computer-aided analyses system for radiographic testing can improve the accuracy of evaluation greatly and overcome the shortcoming of manual evaluating, and make the evaluating process and its results being objective, reasonable and normative. Researchers have done extensive work on welding defects evaluation in radiographic testing.

Suhaila Abd Halim et al. [1] has extracted the weld by converting the image into binary form. The defect boundary is detected by recognizing the black pixel of eight neighborhoods of $3 \times 3$ filtering. The coordinates of the boundary pixel are stored and used to calculate the information of defect features. The information can be used by interpreter to interpret a defect. Abdelhak Mahmoudi et al. [2] proposed a new method for segmenting digitized radiographic images which is based on histogram analysis, contrast enhancement and image thresholding. A region-based hierarchical detection approach is proposed by Ge Liling et al. [3] based on multi-scales segmentation strategies. The proposed method consists of two major stages: initial partition and iterative refinement until all defects are found out. N. Nacereddine et al. [4] developed a method where a set of invariant geometrical attributes which characterize the defect shape. Principal Component Analysis and Artificial Neural Networks are used for weld defect classification. The proposed classification emphasizes in assigning the principal types of weld defects to four categories according to the morphological characteristics of the defects usually met in practice. Aicha Baya Goumeidane et al. [5] developed a new method for automatic estimation of the contours of weld defect in radiographic images. The method uses a statistical formulation of contour estimation by exploiting a region based maximum likelihood criteria. Zhang xiao-guang [6] presents a method of automatic recognition of weld defects based on fuzzy neural network (FNN). The weld image is preprocessed to extract defect features, according to which 8 characteristic parameters are selected, and the FNN model used for defects recognition is set up. To locate the defects and its dimensions in the corresponding weld piece radiographic film image, Arulmozhi.N et al. [7] has compared the acquired radiographic weld image at three different resolutions and denoise those images using non orthogonal log-gabor wavelet function. Abdelhak Mahmoudi et al. [8] presented a new method of segmentation of digitized radiographic images of welds which is based on threshold technique. He compared it with a multiple threshold and Support Vector Machines based method. Zheng Sun et al. [9] proposes a new detection method using fuzzy support vector machine (FSVM) and Beamlet transform. A.B. Goumeidanea et al. [10] present a new approach to deal with the defects contours estimation problem in radiographic images using parametric active contours. Faiza Mekhalfa et al. [11] unsupervised algorithm for radiographic image segmentation, based on the Gaussian mixture models (GMMs). Vijay R.Rathod et al. [12] has proposed presents Analysis and assessment of welding 
images and different techniques involved in detection of flaws in weldments, The efficient and reliable detection of defects is one of the important Task in Nondestructive testing (NDT) And Nondestructive evaluation (NDE). The present paper is organized as follows. Section II deals with the methodology and Section III describes results and discussions followed by conclusions at Section IV.

\section{METHODOLOGY}

Radiography is the mostly used technology adapted in nondestructive techniques (NDT) due to its simplicity and implementation speed. The increased use of radiography as inspection tool are due to the film cost, environmental aspect and safety factors in which less exposure time is applied to produce the image. Welding is a process of joining several pieces of metal. Improper welding procedure may produce defects. Defect is any flaw that is also known as discontinuity of product that is below the acceptance standards. After digitizing the films, it is common practice to adopt a preprocessing stage for the images with the specific purpose of reducing/eliminating noise and improving contrast. Radiographic films usually have noise and deficient contrast due to the intrinsic factors involved in the inspection technique, such as non-uniform illumination and the limited range of intensities of the image capture device. The wiener filter can be defined as a Mean Squared Error optimal stationary linear filter for images degraded by additive noise and blurring. It has to be presumed that the signal and noise processes are second-order stationary (in the random process sense), for the computation of wiener filtering. The Wiener filtering is a linear estimation of the original image. It is simple to see that the Wiener filter has two separate parts, an inverse filtering part and a noise smoothing part. It not only performs the deconvolution by inverse filtering (high pass filtering) but also removes the noise with a compression operation (low pass filtering). So, the present paper first applies an adaptive 7-by-7 Wiener filter for reducing/eliminating noise in the input radiographic image.

From a general scientific perspective, the word morphology refers to the study of form and structures. Mathematical morphology enables to make several measurements on the image. Morphology is referred as a tool for extracting image components that are useful in the representation and description of region shape, such as boundaries, skeletons, and the convex hull like. The initial form of mathematical morphology is applied to binary images and usually referred to as standard mathematical morphology in the literature in order to be discriminated by its later extensions such as the gray-scale and the soft mathematical morphology. A morphological operation analyzes and manipulates the structure of an image by marking the locations where the structuring element fits. Dilation, Erosion, Opening and Closing etc... are some of the morphological operations. Dilation fills the gaps or holes of the image, Erosion widens or enlarges the holes of the image, Erosion followed by closing is called opening and Dilation followed by erosion is called closing. Opening operation decreases the sizes of small, bright details with no appreciable effect on the darker gray levels. The present paper applies morphological opening operation on the filtered image. Then the statistical average value of each pixel is calculated and the segmentation is performed by the Equation 1. Finally the binary image is obtained by performing global thresholding operation. The proposed method for extracting weld defects in radiographic films is given by the following algorithm.

Algorithm:

Step 1: Read the input image of size $\mathrm{M} \times \mathrm{N}$ size

Step 2: Apply the wiener filter on the input image

Step 3: Morphological opening operation is applied on the result of step 2. Where the opening operation can separate the objects that are connected in the Image. Let the Resultant image as $\mathrm{O}$.

Step 4: For each pixel at location $(\mathrm{i}, \mathrm{j})$, compute the average value of all its 9 neighborhood pixel values in $\mathrm{O}$. The neighbor pixels are represented in Fig. 1.

\begin{tabular}{|c|c|c|}
\hline$(\mathrm{i}-1, \mathrm{j}-1)$ & $(\mathrm{i}-1, \mathrm{j})$ & $(\mathrm{i}-1, \mathrm{j}+1)$ \\
\hline$(\mathrm{i}, \mathrm{j}-1)$ & $(\mathrm{i}, \mathrm{j})$ & $(\mathrm{i}, \mathrm{j}+1)$ \\
\hline$(\mathrm{i}+1, \mathrm{j}-1)$ & $(\mathrm{i}+1, \mathrm{j})$ & $(\mathrm{i}+1, \mathrm{j}+1)$ \\
\hline
\end{tabular}

Fig. 1 Representation of neighbor pixels for pixel at location $(i, j)$.

Step 5: For each pixel at location $(\mathrm{i}, \mathrm{j})$, calculate the new gray value by the Equation (1).

Newvalue $(i, j)=(4 \times O(i, j))-(3 \times A v g)$

Repeat Step 3 and 4 for all pixels of size $\mathrm{M} \times \mathrm{N}$. Let the resultant image as $\mathrm{N}$.

Step 6: Convert the gray level image $\mathrm{N}$ to binary image by using global thresholding method. 


\section{RESULTS AND DISCUSSIONS}

The proposed algorithm is tested on various defective radiographic weld images. In this paper the results of cluster porosity (CP), slag inclusion (SI), lack of penetration (LoP), inadequate weld reinforce (IWR) and oxide inclusion (OI) weld defective images are presented. The stepwise results of the proposed method for defect detection are shown in Fig. 2 to 6 . The results clearly indicate that by applying wiener filter, noise is removed and the dark portions are enhanced in the input image. By the second step o applying opening morphological operation, fine interior segments are identified and the overlapped regions are detected by the statistical operation in step 4. Finally by the step four, the defects in the input weld image are given by the binary image computed by global threshold method.

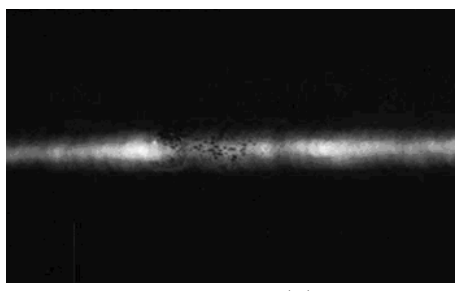

(a)

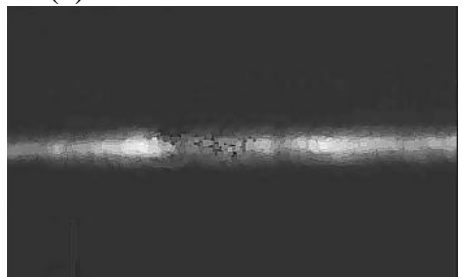

(d)

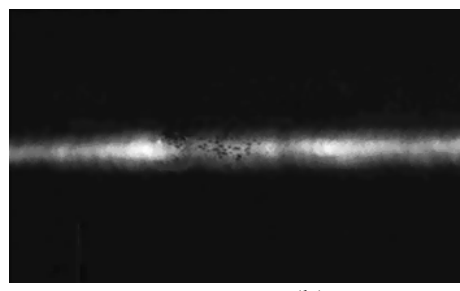

(b)

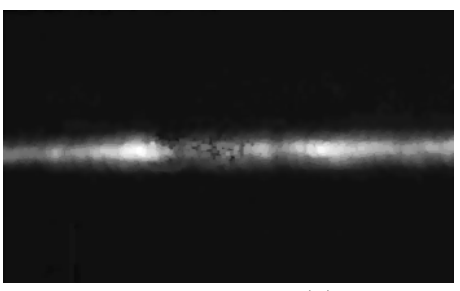

(c)

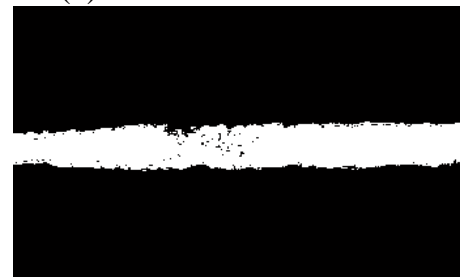

(e)

Fig. 2 Step by step results of the proposed defect detection algorithm (a) original image of CP defect (b) Weiner Filter (c) Opening (d) Statistical (e) Binary image.

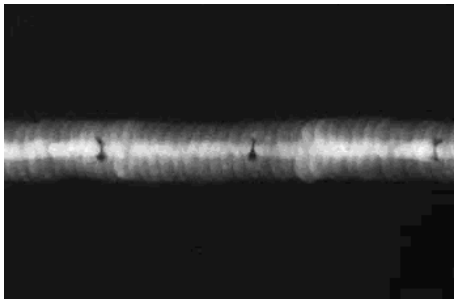

(a)

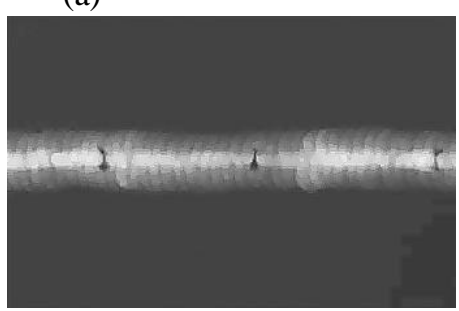

(d)

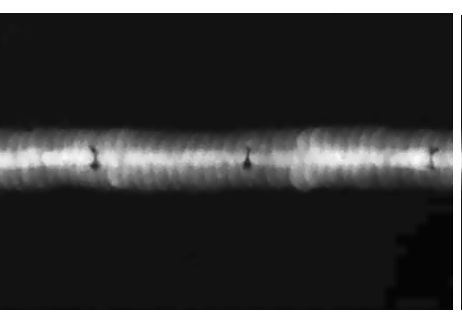

(b)

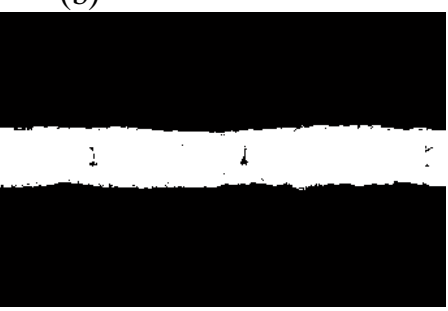

(e)

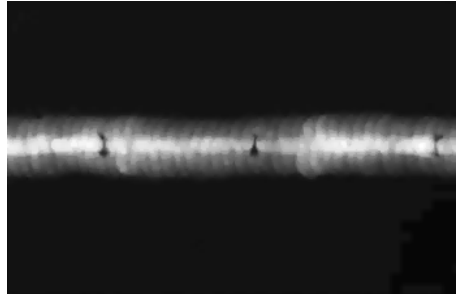

(c)

Fig. 3 Step by step results of the proposed defect detection algorithm (a) original image of SI defect (b) Weiner Filter (c) Opening (d) Statistical (e) Binary image.

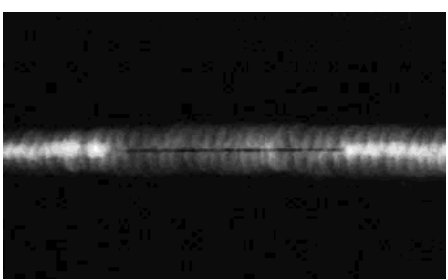

(a)

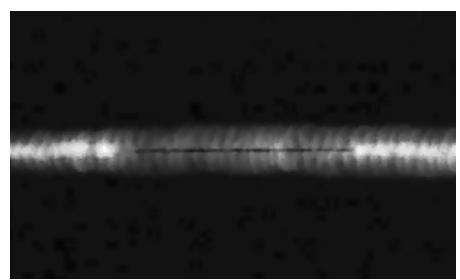

(b)

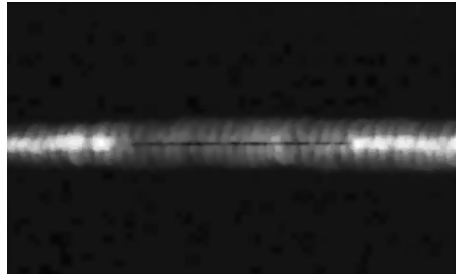

(c) 


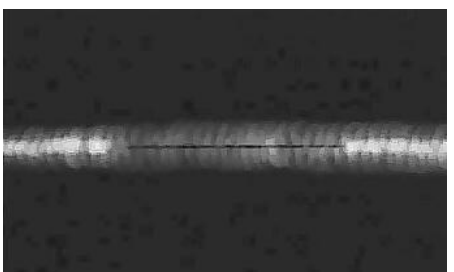

(d)
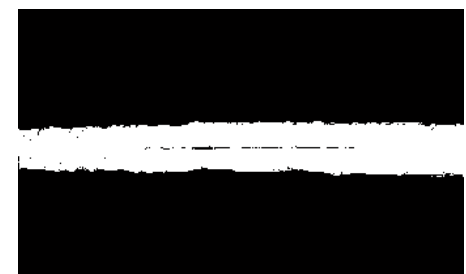

(e)

Fig. 4 Step by step results of the proposed defect detection algorithm (a) original image of LoP defect (b) Weiner Filter (c) Opening (d) Statistical (e) Binary image.

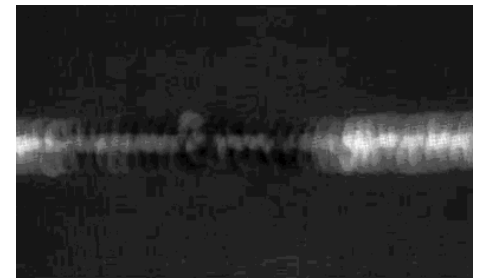

(a)

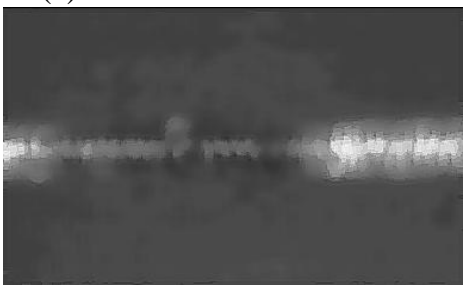

(d)

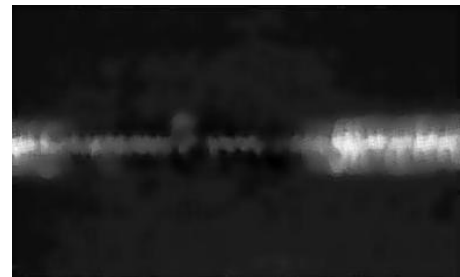

(b)

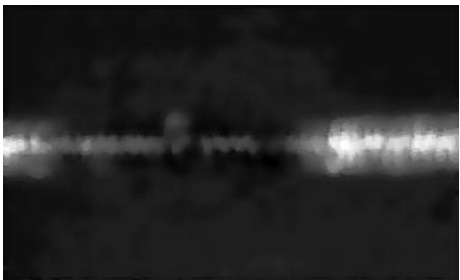

(c)

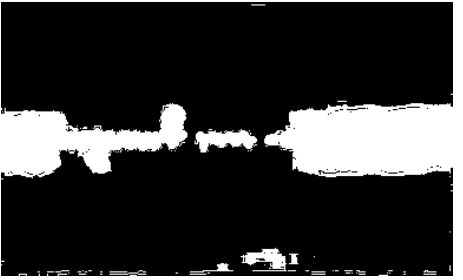

(e)

Fig. 5 Step by step results of the proposed defect detection algorithm (a) original image of IWR defect (b) Weiner Filter (c) Opening (d) Statistical (e) Binary image.

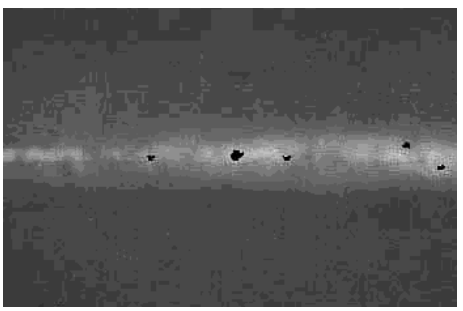

(a)

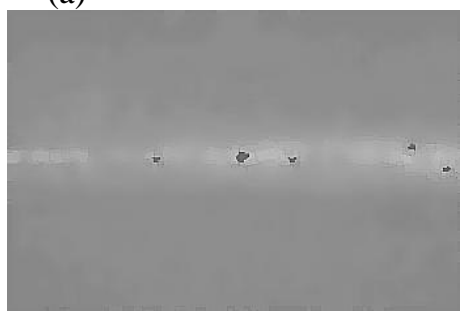

(d)

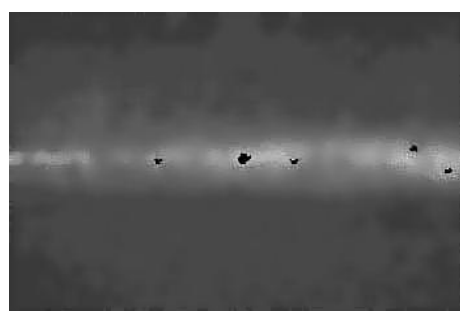

(b)

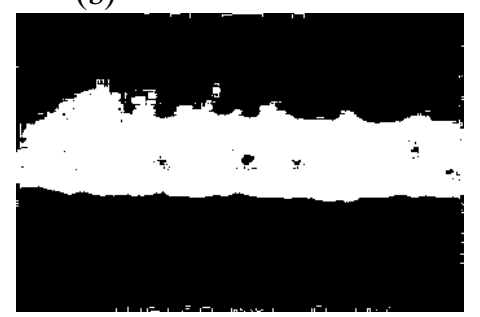

(e)

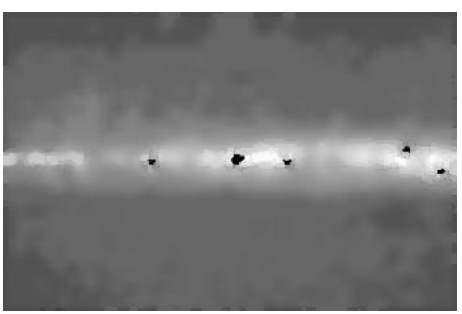

(c)

Fig. 6 Step by step results of the proposed defect detection algorithm (a) original image of OI defect (b) Weiner Filter (c) Opening (d) Statistical (e) Binary image.

\section{CONCLUSIONS}

The proposed method automatically detected various types of defects in radipgraphic weld images by applying wiener filter, morphological opening, statistical and global threshold operations. The experimental results show the effectiveness of the proposed method to detect the defects. The future work will focus on classifying various defective weld images. 


\section{REFERENCES}

[1] Suhaila Abd Halim, Normi Abd Hadi, Arsmah Ibrahim, Yupiter HP Manurung, "The Geometrical Feature of Weld Defect in Assessing Digital Radiographic Image”, IEEE.

[2] Abdelhak Mahmoudi \& Fakhita Regragui, "Welding Defect Detection by Segmentation of Radiographic Images", IEEE Conference on World Congress on Computer Science and Information Engineering, 2009, pp. 111-115.

[3] Ge Liling and Zhang Yingjie, "Hierarchical Segmentation Approach to Detection of Defects on Welding Radiographic Images", IEEE ICIEA, 2009, pp. 2089-2094.

[4] N. Nacereddine and M. Tridi, "Computer-aided shape analysis and classification of weld defects in industrial radiography based invariant attributes and neural networks", Proceedings of the IEEE 4th International Symposium on Image and Signal Processing and Analysis, 2005, pp. 88-93.

[5] Aicha Baya Goumeidane, Mohammaed Khamadja and Nacereddine Nafaa, "Statistical Deformable Model Based Weld Defect Contour Estimation in Radiographic Inspection”, CIMCA, IAWTIC and ISE, 2008, pp. 420- 425.

[6] ZHANG xiao-guang, XU jian-Jan, LI yu, "The Research of Defect Recognition for Radiographic Weld Image Based on Fuzzy Neural Network", Proceedings of the 5n World Congress on Intelligent Control, and Automation, June 15-19, 2004, Hangzhou, P.R. China, pp. 2661-2665

[7] Arulmozhi.N, Manoharan. N,Sheela Rani.B, Venkatraman.B, Baldev Raj, "Isolation of Defects in Radiographic Weld Images with Wavelet Denoising using Log-Gabor filter", IEEE International Conference on Computational Intelligence and Multimedia Applications, 2007, pp. 394-399.

[8] Abdelhak Mahmoudi and Fakhita Regragui, "A Fast Segmentation Method for Defects Detection in Radiographic Images of Welds", IEEE, 2009, pp. 857-860.

[9] Zheng Sun, Dianxu Ruan, Yun Ma, Xiaolei Hu, Xiao-guang Zhang, "Crack Defects Detection in Radiographic Weldment Images using FSVM and Beamlet Transform”, IEEE Sixth International Conference on Fuzzy Systems and Knowledge Discovery, 2009, pp. $402-406$.

[10] A.B. Goumeidane, M. Khamadja, C. Odet, "Parametric Active Contour for Boundary Estimation of Weld Defects in Radiographic Testing", IEEE 2007.

[11] Faiza Mekhalfa, Nafaa Nacereddine and Aïcha Baya Goumeïdane, "Unsupervised Algorithm for Radiographic Image Segmentation Based on the Gaussian Mixture Model", IEEE EUROCON The International Conference on "Computer as a Tool" Warsaw, September 9-12, 2007, pp. 289-293.

[12] Vijay R.Rathod, Alaknanda, R. S. Anand, "Comparison of Different Segmentation Techniques in Detection of Flaws in Weldments", IEEE, 2010, pp. 673-677. 